\title{
Aportes desde la universidad pública a la construcción de paz: prácticas formativas de Terapia Ocupacional y Fonoaudiología con un grupo de personas en proceso de reincorporación en Cali, Colombia
}

Contributions from the public university to the construction of peace: Occupational Therapy and Speech Therapy training practices with a group of people in process of reinstatement in Cali, Colombia

Contribuições da universidade pública para a construção da paz: práticas de treinamento em Terapia Ocupacional e Fonoaudiologia com um grupo de pessoas em processo de reincorporação em Cali, Colômbia

Melania Satizabal Reyes ${ }^{1}$

Andrea Aguilar Arias ${ }^{2}$

Recibido: 9 de abril 2019 • Enviado para modificación: 20 de diciembre 2019 • Aceptado: 21 de enero 2020

Satizábal-Reyes, M. y Aguilar-Arias, A. (2019). Aportes desde la universidad pública a la construcción de paz: prácticas formativas de Terapia Ocupacional y Fonoaudiología con un grupo de personas en proceso de reincorporación en Cali, Colombia. Revista Ocupación Humana, 19 (2), 73-85. https://doi.org/10.25214/25907816.865

\section{RESUMEN}

En el marco de la firma del Acuerdo de Paz entre el Gobierno colombiano y las Fuerzas Armadas Revolucionarias de Colombia - Ejército del Pueblo (FARC-EP), la Universidad del Valle implementó un programa que promueve el desarrollo de prácticas profesionales. Esto permitió a docentes y estudiantes de Terapia Ocupacional y Fonoaudiología elaborar una propuesta que se desarrolló en la ciudad de Cali con la población en proceso de reincorporación y sus familias, como parte del convenio entre la Universidad y la Agencia para la

1 Terapeuta ocupacional. Especialista en Desarrollo Comunitario. Magíster en Sociología. Profesora, Escuela de Rehabilitación Humana, Universidad del Valle. Cali, Colombia. melania.satizabal@correounivalle.edu.co iD http://orcid.org/0000-0003-0793-6031

${ }^{2}$ Fonoaudióloga. Magíster en Epidemiología. Profesora, Escuela de Rehabilitación Humana, Universidad del Valle. Cali, Colombia. andrea.aguilar@correounivalle.edu.co iD https://orcid. org/0000-0002-2146-1741 
Reincorporación y la Normalización, durante el primer semestre del 2018. La experiencia tuvo el propósito de favorecer los procesos de lectura y escritura en los participantes, promoviendo su participación social y la construcción de ciudadanía. La propuesta privilegió un enfoque de construcción conjunta. Participaron diez personas adultas y seis infantes. Se realizaron cinco encuentros, en los cuales se identificaron necesidades e intereses relacionados con leer y escribir, y se construyeron de manera conjunta actividades de promoción de la lectura y la escritura a través de la poesía, el cuento y el teatro. La propuesta posibilitó un ejercicio de reconocimiento y resignificación mutua que permite la construcción de ciudadanía y de profesionales como sujetos políticos.

\title{
PALABRAS CLAVE
}

paz, Terapia Ocupacional, Fonoaudiología, universidad pública, servicio universitario

\begin{abstract}
Within the framework of the signing of the Peace Agreement between the Colombian Government and the Revolutionary Armed Forces of Colombia - People's Army (FARC-EP), Universidad del Valle implemented a program that promotes the development of professional practices, which allowed the preparation of a proposal from teachers and students of Occupational Therapy and Speech Therapy, which was carried out within the framework of the agreement with the Agency for Reincorporation and Standardization. It was carried out in the city of Cali with the population in the process of reinstatement and their families during the first half of 2018 with the purpose of supporting the reading and writing processes in the participating population, thus favoring their social participation and the construction of citizenship. The proposal favored a joint construction approach among all involved. 10 adults and 6 children participated. Five meetings were held which allowed identifying the needs and interests of the participants in relation to reading and writing, and jointly build activities that promoted reading and writing through poetry, storytelling and theater. The proposal enabled an exercise of recognition and mutual resignification that allows the construction of citizenship and professionals as political subjects.
\end{abstract}

\section{KEY WORDS}

peace, Occupational Therapy, Speech Therapy, public education, study service (higher education)

\section{RESUMO}

No âmbito da assinatura do Acordo de Paz entre o Governo da Colômbia e as Forças Armadas Revolucionárias da Colômbia - Exército Popular (FARC-EP), a Universidad del Valle implementou um programa que promove o desenvolvimento de práticas profissionais. Isso permitiu que professores e alunos de Terapia Ocupacional e Fonoaudiologia elaborassem uma proposta - desenvolvida na cidade de Cali com a população no processo de reincorporação e suas famílias -, como parte do acordo entre a Universidade e a Agência de Reincorporação e Padronização, durante o primeiro semestre de 2018. A experiência teve como objetivo favorecer os processos de leitura e escrita dos participantes, promovendo sua participação social e a construção da cidadania. A proposta privilegiou uma abordagem 
de construção conjunta. Participaram dez adultos e seis meninos. Foram realizados cinco encontros, nos quais foram identificadas necessidades e interesses relacionados à leitura e à escrita, e foram construídas, conjuntamente, atividades para promover a leitura e a escrita através da poesia, contos e teatro. A proposta possibilitou um exercício de reconhecimento e ressignificação mútuos, que permite a construção da cidadania e dos profissionais como sujeitos políticos.

\section{PALAVRAS-CHAVE}

paz, Terapia Ocupacional, Fonoaudiologia, universidade pública, serviço universitário

\section{Introducción}

La firma del Acuerdo Final para la Terminación del Conflicto y la Construcción de una Paz Estable y Duradera entre el Gobierno Nacional y las Fuerzas Armadas Revolucionarias de Colombia- Ejército del Pueblo (FARC-EP) marcó un nuevo momento de la historia política y social del país (Oficina del Alto Comisionado para la Paz, 2016). El Acuerdo es coherente con la Constitución Nacional (1991), que proclama "la paz como un derecho fundamental y un deber de obligatorio cumplimiento" (art. 22) e implica el concurso de toda la ciudadanía para "defender y difundir los derechos humanos como fundamento de la convivencia pacífica (...) y propender al logro y mantenimiento de la paz" (art. 95).

En este contexto del posacuerdo y de la construcción de paz, estamos convocados para hacer posible un nuevo país desde el lugar que nos corresponde; personas, grupos e instituciones debemos comprometernos y aportar decididamente a la construcción de una sociedad más justa, que garantice los derechos fundamentales para que todas y todos en Colombia podamos vivir en paz (Franco, 2017). Una de las instituciones Ilamadas a aportar en el proce- so es, justamente, la academia, la universidad; quienes hacemos parte de la educación superior pública en Colombia tenemos el compromiso ineludible de defender el Acuerdo de Paz, estamos Ilamados a ser garantes de su cumplimiento con miras a posibilitar un país distinto para las nuevas generaciones. Como quedó consignado en la Carta de los 472 académicos por la paz: "este momento histórico (...) surge como el más importante para construir una nueva noción de ciudadanía inclusiva e incluyente y proteger, respetar y garantizar los derechos humanos después de más de 50 años de guerra" (Cartas de los lectores, 2017).

La universidad pública, reconociendo su papel como actor clave en la formación de profesionales para aportar a la construcción de un nuevo país, se comprometió públicamente con el proceso de paz en Colombia al incluir en la Declaración del Sistema Universitario Estatal - SUE el punto 9: “apoyo a las prácticas universitarias estudiantiles para la paz", con las cuales se espera, por una parte, que los estudiantes reconozcan las realidades de los territorios y que, además, se comprometan con la 
generación de propuestas para aportar a la construcción de paz (SUE, 2016).

La Universidad del Valle, comprometida con la región Suroccidente y, a través de su Proyecto Institucional, con la construcción de paz y la solución pacífica de conflictos, implementó por medio de la Resolución No.1970 (2016) el programa institucional denominado Educación para la Convivencia, Reconciliación, Derechos Humanos, Cultura de Paz y Posconflicto en Colombia, que para efectos de este texto será referido como programa institucional de paz.

El programa institucional de paz de la Universidad del Valle promueve el desarrollo de actividades de formación, investigación y proyección social, que aporten a la construcción de la paz y la reconciliación en el contexto del posconflicto en los territorios. Una de las líneas de trabajo del programa institucional de paz es la de intervenciones para la paz, en la cual convergen diversas propuestas que van desde el acompañamiento en terreno a comunidades, instituciones y poblaciones vinculadas al conflicto armado, hasta acciones que impactan internamente la construcción de paz en el campus universitario. En las intervenciones, además de las poblaciones víctimas y excombatientes, participan profesores, estudiantes, trabajadores y voluntarios (Universidad del Valle, 2018).

Desde el 2017, un grupo de profesores, con el apoyo del director del programa institucional de paz, ha venido trabajando en la consolidación de una línea o eje de trabajo en torno a las prácticas profesionales para la paz. Desde allí se gestó una iniciativa pionera de prácticas estudiantiles de salud y rehabilitación, la cual se concretó con la elaboración y la ejecución de la propuesta de práctica de estudiantes de último semestre de Terapia Ocupacional y Fonoaudiología de la Universidad.

En este artículo se relata una experiencia piloto de prácticas de rehabilitación para la paz liderada por dos profesoras y un grupo de estudiantes de último año de los programas de Terapia Ocupacional y Fonoaudiología de la Universidad del Valle. Se trata de la implementación de la propuesta de intervención denominada Lectura y Escritura para la Población Excombatiente y sus Familias, que tuvo como objetivo favorecer los procesos de lectura y escritura en población excombatiente y sus familiares, promoviendo así su participación social y la construcción de ciudadanía. Esta fue una de las intervenciones realizadas desde las prácticas estudiantiles en la ciudad de Cali durante el primer semestre del 2018, en el marco del convenio interinstitucional de prácticas y pasantías celebrado entre la Universidad de Valle y la Agencia para la Reincorporación y la Normalización (ARN).

\section{Relato de la experiencia}

\section{Los puntos de partida}

En el segundo semestre del 2017, dos profesoras, una de Terapia Ocupacional y una de Fonoaudiología, emprendimos por decisión propia la búsqueda de oportunidades, desde la Universidad, para realizar acciones que contribuyeran al momento cumbre que vivía el país: la puesta en marcha de la implementación del Acuerdo de Paz entre el Gobierno y las FARC-EP. Fue entonces 
cuando nos acercamos al programa institucional de paz y se abrió el camino para plantear ideas, construir propuestas y realizar alianzas.

La primera alianza fue con un profesor del Instituto de Psicología, a través suyo se logró la articulación a la propuesta denominada Proyecto de Vida, que venía desarrollando como parte del convenio entre la Universidad y la Agencia para la Reincorporación y Normalización - Valle. La propuesta tenía como objetivo acompañar a un grupo de excombatientes en la identificación de herramientas personales y sociales para la construcción de su proyecto vida. Esta articulación nos permitió establecer vínculos con los profesionales de la Agencia encargados del proceso de reincorporación en el departamento del Valle del Cauca y en el norte del departamento del Cauca, y conocer, a través de ellos, las necesidades e intereses de los excombatientes, recuperando así las voces de actores clave en su tránsito a la vida civil.

Desde el inicio, la propuesta de práctica se concibió como un trabajo conjunto que tuviera en cuenta las voces de los actores involucrados para avanzar en el desarrollo de la iniciativa, reconociendo los saberes, las fortalezas y las posibilidades de acción y de toma de decisión de cada quién. Fue así como emergió el tema de leer y escribir. En el desarrollo de la propuesta Proyecto de Vida, los excombatientes manifestaron su interés en retomar o iniciar ocupaciones relacionadas con estudiar y con poner en marcha proyectos productivos, actividades que implican leer y escribir. Por su parte, los profesionales de la Agencia para la Reincorporación y la Normalización manifestaron que los asuntos relacionados con la lectura y la escritura eran una necesidad sentida por los excombatientes para afrontar el tránsito y la reincorporación a la vida civil, particularmente en contextos urbanos.

Es importante plantear que leer y escribir cumplen una función social y política en la vida de los sujetos. Constituyen una forma de comunicación que facilita el acceso a espacios de ciudadanía, pues el uso del código es una exigencia para participar en actividades cotidianas como leer las rutas de los buses o firmar un documento, y en otras más complejas como, por ejemplo, presentar una hoja de vida, celebrar un contrato, seguir las instrucciones para el proceso de admisión en una institución educativa, comprender los textos de las pruebas formales para acceder a la educación superior o elaborar una propuesta o un proyecto productivo; situaciones que van en pro de la consolidación de los proyectos de vida y la resignificación de las ocupaciones de los excombatientes.

Entre tanto, desde la perspectiva profesional de Terapia Ocupacional y Fonoaudiología, durante una jornada de salud dirigida a la población excombatiente y sus familias, realizada en el marco de lo acordado entre la Universidad, la Agencia para la Reincorporación y la Normalización y la población en proceso de reincorporación radicada en Cali, identificamos necesidades respecto al uso formal de la lectura y escritura. Dicha jornada se desarrolló a través de estaciones para la valoración integral de acuerdo con el ciclo de vida, a cargo de docentes y estudiantes de Terapia Ocupacional y Fonoaudiología. En la estación orientada a la primera infancia y a los niños y niñas en edad escolar identificamos alertas tempranas relacionadas 
con aspectos psicomotores, cognitivos y del lenguaje de los participantes. En la estación diseñada para valorar a jóvenes y adultos realizamos un tamizaje de habilidades motrices, cognitivas, sociales y comunicativas. Esto nos permitió identificar aspectos relacionados con la lectura y la escritura, e invitar a los excombatientes y sus familias a participar de la propuesta.

\section{Diseño y concertación de la propuesta}

Una vez identificadas las necesidades e intereses de la población, diseñamos una propuesta piloto con el grupo de estudiantes. Para ese momento, la población excombatiente iniciaba su reincorporación, de manera que presentamos y discutimos esa propuesta con los líderes del proceso (representantes de las FARC en el Valle del Cauca y profesionales de la Agencia), con el objetivo de revisar en conjunto su pertinencia y viabilidad. En coherencia con los principios de la reincorporación, se acordó la participación de los excombatientes y de sus familias; así mismo, respondiendo a una solicitud explícita del equipo de FARC, se planteó la articulación de una de sus integrantes, quien en ese momento hacía parte del comité de educación para la reincorporación de los excombatientes, en la planeación, la ejecución y la discusión de las actividades.

La convocatoria para que la población participara de la propuesta estuvo a cargo de los profesionales de la Agencia para la Reincorporación y la Normalización, siguiendo sus protocolos y cumpliendo así con el papel de mediadora y acompañante que le correspondía en ese momento. Junto con los participantes y la Agencia acordamos desarrollar las actividades los sábados en la mañana en las instalaciones de esta última, ubicadas en el oriente de Cali.

\section{Implementación de la propuesta}

La propuesta se estructuró de manera que los excombatientes pudieran participar con sus familias; para ello, conformamos dos grupos de trabajo: uno para adultos y otro para escolares, con sus respectivos diseños metodológicos y ajustes razonables de las actividades, de acuerdo con las necesidades de los participantes.

Llevamos a cabo cinco encuentros, con una participación promedio de diez excombatientes en cada uno. El primero de ellos, orientado a identificar intereses y necesidades en torno a la lectura y la escritura, se realizó por medio de un conversatorio sobre experiencias a lo largo de la vida, lo que significaban estas actividades para ellos y las necesidades que tenían. En un segundo momento, desarrollamos una actividad de escritura y lectura libre de textos construidos a partir de dos palabras escogidas al azar. Los excombatientes participantes reconocieron la importancia de leer y escribir en la vida cotidiana, en palabras de uno de ellos, "leer y escribir es la riqueza del ser humano"; tambien manifestaron que les permiten compartir con otros sus pensamientos y participar en la sociedad.

Al finalizar el primer encuentro, realizamos una lluvia de ideas sobre actividades relacionadas con leer y escribir en las que los participantes estuvieran interesados; este insumo nos permitió construir, junto con los estudiantes, la planeación de las siguientes sesiones. 
En el segundo encuentro trabajamos alrededor del género lírico, por medio de textos poéticos que leimos y construimos en conjunto con excombatientes, estudiantes y personal de apoyo de la Agencia. En esa ocasión participó también un miembro de la Policía Nacional encargado de apoyar el protocolo de atención en la Agencia. Los participantes sugirieron relacionar la actividad con la celebración del día de la madre, por tanto, los textos estuvieron enfocados en las representaciones que tenían sobre las mujeres y lo femenino. Lograron reconocer la superestructura de textos líricos y construir poemas, además, se generó una reflexión frente a la participación de la mujer en las FARC-EP; manifestaron, por ejemplo: "para nosotros no importaba si quien iba a coordinar era una mujer o un hombre, era cuestión de rangos, tenían igual respeto" o "allá las mujeres cargaban igual que uno".

En el tercer encuentro, abordamos el fomento de habilidades necesarias para leer y escribir, esta vez, a través de la corporeidad. En un juego de mímica se debían representar diferentes ocupaciones, sentimientos, objetos. Esta actividad permitió a los participantes evidenciar el cuerpo como medio de expresión y comunicación, también hizo posible reconocer parte de las historias de vida de los excombatientes y sus familias, y dimensionar el asunto de leer y escribir de múltiples formas, en esta ocasión, a través del cuerpo.

Para los encuentros cuarto y quinto, trabajamos alrededor del teatro. En el cuarto, construimos una obra de teatro a través de la participación en tres estaciones, en las cuales los participantes elegían acciones, a través de un juego de mímica; personajes, ubicando indu- mentaria a su gusto, y lugares, construyendo en conjunto un pequeño mural en el que cada participante dibujaba sitios significativos en su vida y que serviría luego como contexto para la obra. Posteriormente, en conjunto, creamos la obra de teatro vinculando los diferentes personajes, acciones y lugares.

Como cierre, en el último encuentro se presentó la obra de teatro a otros excombatientes y a algunos profesionales de la Agencia para la Reincorporación y la Normalización. Seguidamente, facilitamos la evaluación y realimentación de la propuesta por parte de los participantes, quienes expresaron su gratitud por acompañarlos en el proceso de reincorporación; en palabras de uno de ellos, "gracias por no olvidarnos". Finalmente, entregamos a los participantes un cuadernillo que recopilaba los productos y creaciones generados en cada encuentro, así como rutas de acceso a espacios de arte y cultura en la ciudad que posibilitan leer y escribir en familia.

Por otro parte, realizamos dos encuentros con un grupo promedio de seis niños y niñas en edad escolar, hijos de excombatientes participantes. En el primer encuentro, identificamos sus necesidades e intereses en torno a la lectura y a la escritura. Se hizo la lectura de cuentos en voz alta y les motivamos a crear finales alternativos por medio de la escritura, el dibujo o la narración, de acuerdo con sus habilidades particulares. Lograron involucrarse con estas propuestas y algunos manifestaron necesidades de apoyo para el desarrollo de actividades en el ámbito escolar.

El segundo encuentro estuvo dirigido a promover la creación de historias de manera colectiva. Los niños y niñas 
debían elegir una serie de palabras de diversas categorías semánticas y gramaticales, con el fin de incluirlas en las narraciones. Lograron vincularse al trabajo en grupo y construir historias con sentido, ubicando los personajes, las situaciones y los lugares.

\section{Discusión}

La implementación de la propuesta nos permitió cumplir sus objetivos como fin y como medio. Como fin, en la medida que se pudo establecer una alianza de trabajo con la Agencia para la Reincorporación y la Normalización y las FARC-EP para dar respuesta a una necesidad puntual en un momento inicial del proceso de reincorporación, lo que ha permitido dar curso a otras propuestas.

Además, el uso de estrategias vinculadas con el arte (poesía, teatro, cuento) posibilitó que la interacción y la vinculación con los excombatientes fuese más fluida, cercana, sin temores. Para este primer momento de encuentro con la población, ello resultó muy efectivo, dado el estigma social que encarnan.

Los juegos teatrales promovieron la expresión de sus historias de vida, pero lo más importante es que permitieron como ocurre en otras experiencias que utilizan estas técnicas - que los excombatientes y sus familiares tuvieran voz y un lugar en el tejido de relaciones que ocurrieron alrededor de las actividades planteadas (Justa y de Holanda, 2012; Alves et al., 2013).

Como medio, la implementación de la propuesta posibilitó una serie de reflexiones, entre ellas, que la universidad es ante todo un escenario de construcción de ciudadanía. En el entramado de relaciones sociales que allí se dan, salta a la vista el rol que la sociedad le ha asignado, de consolidar los procesos formativos tendientes a la producción y reproducción de saber, cultura y sociedad. La universidad no se reduce a los procesos de enseñanza y aprendizaje que suceden en sus aulas; ni siquiera se limita estrictamente a la funciones misionales de formación, investigación y extensión; va más allá, es constructora de sociedad. Y lo es aún más la universidad pública, por su misma configuración. Es deber de la universidad pública en Colombia asumir con altura el compromiso de aportar a la conformación "de nuevas expresiones de sociedad, de cultura, de relaciones sociales, de economía, de globalidad, de movimientos y cambios locales intensos" (Didriksson, 2008, p.79).

La universidad pública colombiana no ha sido ajena al conflicto social que vive el país. Los grandes debates en torno a la vida política han pasado por ella de diversas maneras, también lo han hecho las reflexiones y acciones en torno a la paz. Por ello, las iniciativas emprendidas como universidad en torno al trabajo con excombatientes de las FARC-EP significan una recuperación de su papel en la construcción de paz y, a su vez, permiten rescatar el papel político del ejercicio profesional.

La intervención adelantada en el contexto de la práctica profesional de Terapia Ocupacional y Fonoaudiología es, ante todo, una acción política de construcción de ciudadanía en doble vía: por una lado, la población excombatiente que se incorpora a la vida civil $y$, por el otro, el conjunto de profesio- 
nales en formación que resignifican, en clave de reconciliación, a uno de los actores del conflicto armado en Colombia.

Es una acción política, además, porque implicó un ejercicio de construcción colectiva que pasa por el reconocimiento. Reconocemos la existencia de un conflicto con sus causas, sus actores y sus consecuencias. Reconocemos el acto político de firmar un acuerdo de paz y sus implicaciones para la construcción de un nuevo país. Reconocemos las subjetividades de excombatientes enfrentados a la tarea de redireccionar sus proyectos de vida y sus ocupaciones, sin la mediación de la estructura armada y sus dinámicas.

Reconocemos, también, el nacimiento de nuevos escenarios para la universidad, ahora con la presencia de excombatientes de las FARC-EP. En ese sentido, el ejercicio de la docencia universitaria en la Colombia del posacuerdo requiere nuevas prácticas pedagógicas acordes con la construcción de una sociedad que empieza a pensarse desde la paz, no desde la violencia.

Este ejercicio de reconocimiento y transformación mutua es parte de esa construcción de ciudadanía y de profesionales como sujetos políticos (García, 2016) que le compete a la universidad y que, como universidad, contribuimos a materializar con las acciones desde las prácticas pedagógicas (de las profesoras) y académicas (de los estudiantes) relatadas en este artículo.

Queda para la universidad pública el desafío de pensarse en esta tarea, sabiendo que requiere una profunda adecuación de sus estructuras para seguir facilitando la construcción de paz y de sociedad. Para que la universidad siga siendo crítica, incluyente, transformadora, debe ser capaz de mirarse a sí misma y a la sociedad más allá de lo puramente académico y de lo estrictamente disciplinar, para garantizar el debate, el disenso y, finalmente, el consenso sobre lo básico, sobre lo fundamental: la tramitación pacífica de los conflictos y la construcción de una sociedad más justa, más equitativa y en paz. La apertura de procesos y puertas es precedida por una apertura de mente; en este caso, tal apertura se dio $y$, en la medida en que esto siga ocurriendo, la universidad continuará siendo protagonista en la consolidación de una sociedad en paz para Colombia.

La práctica posibilitó reconstruir fragmentos de la memoria del conflicto desde las voces de uno de los actores; en esa medida, promovió la reconciliación, un factor determinante en la transformación de las prácticas culturales en los procesos de paz. Permeó además a una generación que ha conocido la historia del conflicto desde la ciudad. Posibilitó, tanto a estudiantes como a las profesoras, relacionarnos con los excombatientes desde un lugar diferente al del estigma. En palabras de una estudiante, "la práctica me permitió entender que los excombatientes son personas como nosotros... es que uno se los imagina, no sé, diferentes... y al final, con el trato, uno se da cuenta que son colombianos con otra historia de vida... eso es".

Dentro de las limitaciones de la implementación de la propuesta podemos enunciar, en primera instancia, que los imaginarios negativos sobre los excombatientes en el momento del desarrollo de la propuesta, por parte de algunos sectores académicos, sociales y políticos del país, permeó en algún grado el 
inicio del proyecto. Esto llevó, incluso, a cuestionamientos desde instancias académicas sobre nuestra participación como profesoras en el proceso. También hubo retos constantes en relación con colegas docentes y no docentes, estudiantes y familiares de estudiantes, quienes manifestaron temores e incertidumbres por el trabajo con excombatientes de las FARC-EP desde las prácticas.

De otro lado, la baja e irregular asistencia de los excombatientes a las sesiones de trabajo, así como la necesidad de cambiar con frecuencia los horarios acordados para los encuentros, nos exigieron como equipo un alto grado de flexibilidad y creatividad para lograr las metas propuestas. Entre las razones para estos cambios, algunos participantes reportaron que se encontraban ajustando sus rutinas en la ciudad, lo que comprometía su disponibilidad de tiempo.

A su vez, la construcción de confianza entre profesoras, estudiantes y excombatientes tomó tiempo y estuvo mediada, en principio, por las relaciones ya establecidas entre estos últimos y la Agencia encargada de la reincorporación en Cali.

Un último aspecto que generó tensiones al inicio del proyecto fue el diálogo de saberes y la construcción de acuerdos entre profesoras y estudiantes de Terapia Ocupacional y de Fonoaudiología. En parte por la diversidad en tiempos, intensidad y dedicación de profesoras y estudiantes, pero también por los imaginarios, las experiencias y las expectativas de los integrantes del equipo respecto al trabajo con población excombatiente.

Por otra parte, es importante resaltar el papel de ambas profesiones, Terapia Ocupacional y Fonoaudiología, las cuales han estado vinculadas de manera directa e indirecta con investigaciones e intervenciones en el escenario del conflicto armado y el posacuerdo en Colombia.

En el contexto nacional se han documentado algunas experiencias e investigaciones de terapeutas ocupacionales con población víctima del conflicto armado en situación de desplazamiento forzado, quienes viven afectaciones en su bienestar ocupacional. La Terapia Ocupacional ha contribuido en la caracterización ocupacional de la población y en la implementación de estrategias de orientación profesional que permitan la adquisición de nuevos roles, hábitos y rutinas, con la intención de aportar en la consecución de nuevas oportunidades ocupacionales (Gómez et.al., 1999; Tibaduiza et al., 2006).

También se han caracterizado experiencias de terapeutas ocupacionales relacionadas con la superación del conflicto armado en varias regiones del país. Gomez et al. (2017) refieren que la mayor parte de las intervenciones han estado dirigidas a la atención de víctimas del conflicto, población adulta y con discapacidad, principalmente con abordajes comunitarios

Los reportes sobre trabajos relacionados con población victimaria son escasos, encontrándose, en una breve revisión de literatura, solo una propuesta de atención sociolaboral a exguerrilleros en proceso de reinserción (Cuellar, 2000). Esta situación denota un reto para la profesión, en la medida que esta población se encuentra en mayor riesgo de exclusión, derivado del estigma social (Peñas et al., 2015). La experiencia que aquí 
relatamos cobra entonces importancia, dado que contribuye a la documentación del trabajo que desde la profesión se realiza con excombatientes.

En el caso de Fonoaudiología, algunas experiencias en el contexto de las violencias en Colombia dan cuenta, por una parte, de la importancia del lenguaje y la comunicación como elementos centrales en los procesos de inclusión y de reincorporación; por otra, de las discapacidades auditivas como una de las consecuencias directas del conflicto armado en el país. Álvarez (2006), por ejemplo, reportó el caso de niñas y niños campesinos desplazados por violencia y su inclusión en escuelas urbanas, resaltando que la mayor potencia para su adaptación estuvo en el uso del lenguaje como mediador en los procesos de aprendizaje en el aula.

\section{Consideraciones finales}

Implementar la propuesta permitió acercar la Universidad, lo que ella representa, sus docentes y estudiantes, con los excombatientes y sus familias; este acto, por sí solo, hizo posible generar una reparación simbólica con una población que ha decidido dejar las armas y construir un proceso de paz. Por otra parte, fue una oportunidad para repensar y transformar el lugar que tenemos como profesionales de salud, con miras a contribuir a una sociedad más justa y equitativa, que posibilite la implementación de los acuerdos de paz (Rojas, 2017).

La presencia de la Universidad del Valle, a través de las prácticas de profesoras y estudiantes de Terapia Ocupacional y Fonoaudiología con población excombatiente en proceso de reincorporación, permitió hacer aportes desde la salud a la construcción de paz. Salud y paz, como derechos, convergen en un momento en el que el país avanza por la vía de la tramitación del conflicto sin mediación de las armas; eso pasa por la garantía de mejores condiciones de salud para todos. Sin salud no hay paz; sin paz, difícilmente habrá salud (Franco, 2017).

Esta experiencia se puede considerar como un piloto desde la Facultad de Salud de la Universidad del Valle, que abona el camino para que las prácticas de otros programas del área se articulen al proceso. Además, ha impulsado la formulación de otros proyectos, entre ellos, la construcción de rutas de apoyo educativo para la población excombatiente que se encuentra terminando la básica secundaria o iniciando la vida universitaria.

El proceso de reincorporación de excombatientes demanda un ejercicio de resignificación de las profesiones de la salud en general y de la Terapia Ocupacional y la Fonoaudiología en particular. Para la Terapia Ocupacional, resignificar las ocupaciones abre una gama de oportunidades para propuestas alternativas que respondan al nuevo escenario. Para la Fonoaudiología, resignificar el papel del lenguaje en el escenario de la construcción de paz implica redimensionar el alcance de la profesión y generar rupturas con paradigmas que siguen siendo hegemónicos. Queda como reto y compromiso, divulgar las experiencias formativas, profesionales e investigativas que del tema se deriven para contribuir a esa transformación.

La formación política debe ser una constante en la formación y en el ejercicio profesional de terapeutas ocupacio- 
nales y fonoaudiólogos, para lograr incidir en las instituciones encargadas del diseño y la implementación de políticas dirigidas a excombatientes, de manera que se identifique, valore e incluya el perfil de ambas profesiones en los procesos de reincorporación y reintegración de esta población.

La incursión de profesionales de Terapia Ocupacional y de Fonoaudiología

\section{Referencias}

Alves, I., Tavares, G. T., y Castro, H. A. (2013). Teatro do oprimido e Terapia Ocupacional: uma proposta de intervenção com jovens em situação de vulnerabilidade social. Cadernos Brasileiros de Terapia Ocupacional, 21(2), 325 - 337. http://dx. doi.org/10.4322/cto.2013.034

Alvarez, B. (2006). Niños campesinos desplazados por la violencia: una nueva minoría cultural en las escuelas urbanas colombianas. Revista de la Facultad de Medicina, 54(3), 219-224. http://dx.doi. org/10.15446/revfacmed

Asamblea Nacional Constituyente (1991). Constitución Política de la República de Colombia. Gaceta Constitucional № 116. http://www.secretariasenado.gov.co/senado/basedoc/constitucion politica 1991. $\underline{\mathrm{html}}$

Cartas de los lectores (2017, 21 de diciembre). Carta de 472 académicos por la Paz. El Espectador. https://www.elespectador.com/ opinion/carta-de-472-academicos-en-respaldo-la-paz-columna-729746 en el trabajo con personas en proceso de reincorporación implica una comprensión contextual amplia de la realidad sociopolítica del país y de los territorios, y de cómo en ella se entretejen las historias de vida y el devenir ocupacional y social de los excombatientes. Además, implica construir abordajes interdisciplinarios consistentes con la complejidad de estos asuntos.

Cuellar, P. (2000). Una propuesta de atención sociolaboral para ex guerrilleros. Revista Ocupación Humana, 8(3), 24-32. https:// doi.org/10.25214/25907816.158

Didriksson, A. (2008). Contexto global y regional de la educación universitaria en América Latina y el Caribe. En L. Bonilla-Molina y F. López-Segrera (comp.), Educación universitaria para el siglo XXI. Análisis comparados (I) (pp.77-114). Ministerio del Poder Popular para la Educación Universitaria y Centro Internacional Miranda.

Franco, S. (2017). Por la salud social. Aurora.

García, S. (2016). Terapeuta ocupacional: sujeto político. Revista Ocupación Humana, 16(1), 84-90. https://doi. org/10.25214/25907816.16

Gómez, A. M., Peñas, O. L. y Parra, E. I. (2017). Experiencias de Terapia Ocupacional para la paz: aportes desde las regiones colombianas. Revista de Salud Pública, 19(5), 664-670. http://dx.doi.org/10.15446/rsap. v19n5.6248 
Justa, F. M. C. y de Holanda, I. C. L. (2012). Teatro com adolescentes em risco social: práticas de promoção da saúde no contexto terapêutico ocupacional. Revista de Terapia Ocupacional da Universidade de São Paulo, 23(1), 16-23.

Oficina del Alto Comisionado para la Paz (2016). El Acuerdo final de paz. La oportunidad para construir paz. https://www. refworld.org.es/pdfid/5a874f254.pdf

Gómez, M., Torres, S. y García, M (1999). Caracterización del comportamiento ocupacional de personas desplazadas por la violencia. Revista Ocupación Humana, 8(2), 13-31. https://doi. org/10.25214/25907816.483

Peñas, O. L., Gómez, A. M., y Parra, E. I. (2015). Participación de terapia ocupacional en contextos de conflicto armado y postconflicto. Revista de Salud Pública, 17(4), 612-625. http://dx.doi.org/10.15446/rsap. v17n4.53047

Rojas, C. P. (2017). Terapia Ocupacional en el posacuerdo. Retos para una profesión que se transforma. Revista Ocupación Humana, 17(1), 3-6. https://doi. org/10.25214/25907816.150
Sistema Universitario Estatal - SUE (2016). Declaración del Sistema Universitario Estatal - SUE en apoyo al proceso de paz. https://www.mineducacion.gov.co/1759/ w3-printer-357788.html

Tibaduiza, G.M., Lourido, D.C., Criollo, C., y Ortega, M. (2006). Intervención social con población víctima del desplazamientos forzado en el municipio de Ipiales, Nariño, a través de una propuesta de orientación profesional dirigida por el programa de Terapia ocupacional. Revista Unimar, 24(2), 25-29. http://editorial.umariana.edu.co/revistas/index. php/unimar/article/view/9

Universidad del Valle (2016, 4 de mayo). Resolución No. 1970. Por la cual se aprueba el programa institucional, denominado, Educación para la convivencia, reconciliación, derechos humanos, cultura de paz y post conflicto en Colombia. http:// proxse16.univalle.edu.co/ secretariageneral/rectoria/resoluciones/2016/R 1970.pdf

Universidad del Valle (2018). Programa Institucional de Paz. Intervenciones para la Paz. http://programapaz.univalle.edu. co/intervenciones-para-la-paz 